\title{
On Lyapunov-type inequalities for odd order boundary value problems
}

\author{
Mustafa Fahri Aktaş ${ }^{1 *}$, Devrim Çakmak² and Abdullah Ahmetoğlu ${ }^{1}$
}

\section{"Correspondence:}

mfahri@gazi.edu.tr

'Department of Mathematics,

Faculty of Sciences, Gazi University,

Ankara, Turkey

Full list of author information is

available at the end of the article

\begin{abstract}
In this article, we construct new Lyapunov-type inequalities for odd order boundary value problems. The aim of this article is to find the maximum of Green's function $\left|G_{2 n+1}(x, s)\right|$ corresponding to two-point boundary value problems. To the best of our knowledge, there is no paper dealing with Lyapunov-type inequalities for odd order boundary value problems by bounding the Green's function of the same problem. In addition, some applications of the obtained inequalities are also given.
\end{abstract}

MSC: 34C10; 34B15; 34L15

Keywords: Lyapunov-type inequalities; Two-point boundary conditions; Green's functions

\section{Introduction}

In this article, we get new Lyapunov-type inequalities for the $(2 n+1)$ th order problem

$$
\begin{aligned}
& y^{(2 n+1)}+(-1)^{n-1} r(x) y=0, \\
& y^{(k)}\left(x_{1}\right)=y^{(k)}\left(x_{2}\right)=0 \quad \text { for } k=0,1, \ldots, n-1, \\
& (-1)^{n-1} y^{(n)}\left(x_{1}\right)=y^{(n)}\left(x_{2}\right),
\end{aligned}
$$

where $n \in \mathbb{N}, x_{1}, x_{2}$ are real numbers with $x_{1}<x_{2}, r \in C([0, \infty), \mathbb{R})$, and $y$ is a real solution (not identically zero) of (1.1)-(1.3).

In 1907, Lyapunov [1] got an important inequality. If $r \in C([0, \infty), \mathbb{R})$ and $y$ is a solution (not identically zero) of the problem

$$
\begin{aligned}
& y^{\prime \prime}+r(x) y=0, \\
& y\left(x_{1}\right)=y\left(x_{2}\right)=0,
\end{aligned}
$$

then the inequality

$$
\frac{4}{x_{2}-x_{1}} \leq \int_{x_{1}}^{x_{2}}|r(s)| d s
$$

holds. Note that the constant 4 in (1.6) is the best possibility (see [2, p. 345], [3, p. 267]).

(c) The Author(s) 2019. This article is distributed under the terms of the Creative Commons Attribution 4.0 International License (http://creativecommons.org/licenses/by/4.0/), which permits unrestricted use, distribution, and reproduction in any medium, provided you give appropriate credit to the original author(s) and the source, provide a link to the Creative Commons license, and indicate if changes were made. 
With the help of Green's function, Hartman [2] generalized the Lyapunov inequality (1.6) as follows: If $r \in C([0, \infty), \mathbb{R})$ and $y$ is a solution (not identically zero) on $\left[x_{1}, x_{2}\right]$ for problem (1.4)-(1.5), then the inequality

$$
1 \leq \int_{x_{1}}^{x_{2}} \frac{\left(s-x_{1}\right)\left(x_{2}-s\right)}{x_{2}-x_{1}} r^{+}(s) d s
$$

holds, where $r^{+}(x)=\max \{r(x), 0\}$. It is obvious that the function $M(x)=\left(x-x_{1}\right)\left(x_{2}-x\right)$ takes its absolute maximum value at $\frac{x_{1}+x_{2}}{2}$, i.e.,

$$
M(x) \leq \max _{x_{1} \leq x \leq x_{2}} M(x)=M\left(\frac{x_{1}+x_{2}}{2}\right)=\left(\frac{x_{2}-x_{1}}{2}\right)^{2} .
$$

Thus, inequality (1.7) is a natural generalization of inequality (1.6).

In the literature, the authors found some Lyapunov-type inequalities for higher order problems [4-24]. When we look at the work done in the literature, we see that the best Lyapunov constant is obtained by taking the absolute maximum of the Green's functions. Lyapunov-type inequalities for higher order problems by using the Green's function of the same problem can be found in Agarwal and Özbekler [4, 5], Beesack [8], Das and Vatsala [10, 11], and Yang [24]. Moreover, by using Green's functions corresponding to even order boundary value problems, Lyapunov-type inequalities for boundary value problems of order $(2 n+1)$ can be found in Aktaş et al. [7], and Dhar and Kong [12, 13]. To the best of our knowledge there is almost no study about Lyapunov-type inequalities for odd order problem that uses the Green's function of the same problem. Therefore, our aim is to get new Lyapunov-type inequalities for odd order boundary value problem by means of the properties of Green's functions corresponding to the same problem. Before introducing our main results, we remember some important results obtained earlier.

Now, we give the definition of Green's function for two point $n$th order linear boundary value problems.

Definition A ([25, Definition 2.1]) It is said that $G_{n}(x, s)$ is a Green's function for the problem

$$
L_{n} y(x)=0 \quad \text { for } x \in I=\left[x_{1}, x_{2}\right], \quad U_{i}(y)=0 \quad \text { for } i=1,2, \ldots, m,
$$

where

$$
L_{n} y(x) \equiv a_{0}(x) y^{(n)}(x)+a_{1}(x) y^{(n-1)}(x)+\cdots+a_{n}(x) y(x) \quad \text { for } x \in I
$$

and

$$
U_{i}(y) \equiv \sum_{j=0}^{n-1}\left(\alpha_{j}^{i} y^{(j)}\left(x_{1}\right)+\beta_{j}^{i} y^{(j)}\left(x_{2}\right)\right) \quad \text { for } i=1,2, \ldots, m \text { and } m \leq n
$$

being $\alpha_{j}^{i}, \beta_{j}^{i}$ real constants for all $i=1,2, \ldots, m, j=0,1, \ldots, n-1, a_{k}(x)$ is a continuous real function for all $k=0,1, \ldots, n$, and $a_{0}(x) \neq 0$ for all $x$, if it satisfies the following properties:

$\left(G_{1}\right) G_{n}$ is defined on the square $I \times I$. 
$\left(G_{2}\right)$ For $k=0,1, \ldots, n-2$, the partial derivatives $\frac{\partial^{k} G_{n}}{\partial x^{k}}$ exist and they are continuous on $I \times I$.

$\left(G_{3}\right) \frac{\partial^{n-1} G_{n}}{\partial x^{n-1}}$ and $\frac{\partial^{n} G_{n}}{\partial x^{n}}$ exist and are continuous on the triangles $x_{1} \leq x<s \leq x_{2}$ and $x_{1} \leq$ $s<x \leq x_{2}$.

$\left(G_{4}\right)$ For each $x \in\left(x_{1}, x_{2}\right)$, there exist lateral limits $\frac{\partial^{n-1} G_{n}}{\partial x^{n-1}}\left(x, x^{+}\right)$and $\frac{\partial^{n-1} G_{n}}{\partial x^{n-1}}\left(x, x^{-}\right)$(i.e., the limits when $(x, s) \rightarrow(x, x)$ with $s>x$ or with $s<x)$; moreover,

$$
\frac{\partial^{n-1} G_{n}}{\partial x^{n-1}}\left(x, x^{+}\right)-\frac{\partial^{n-1} G_{n}}{\partial x^{n-1}}\left(x, x^{-}\right)=-\frac{1}{a_{0}(x)} .
$$

$\left(G_{5}\right)$ For each $s \in\left(x_{1}, x_{2}\right)$, the function $x \rightarrow G_{n}(x, s)$ is a solution of the differential equation $L_{n} y(x)=0$ on $x \in\left[x_{1}, s\right)$ and $x \in\left(s, x_{2}\right]$. That is,

$$
a_{0}(x) \frac{\partial^{n} G_{n}(x, s)}{\partial x^{n}}+a_{1}(x) \frac{\partial^{n-1} G_{n}(x, s)}{\partial x^{n-1}}+\cdots+a_{n}(x) G_{n}(x, s)=0
$$

on both intervals.

$\left(G_{6}\right)$ For each $s \in\left(x_{1}, x_{2}\right)$, the function $x \rightarrow G_{n}(x, s)$ satisfies the boundary conditions $U_{i}\left(G_{n}(\cdot, s)\right)=0$ for $i=1,2, \ldots, m$ :

$$
\sum_{j=0}^{n-1}\left(\alpha_{j}^{i} \frac{\partial^{j} G_{n}\left(x_{1}, s\right)}{\partial x^{j}}+\beta_{j}^{i} \frac{\partial^{j} G_{n}\left(x_{2}, s\right)}{\partial x^{j}}\right)=0 \quad \text { for } i=1,2, \ldots, m
$$

The main importance of the above definition is that the integral operator, whose kernel is a Green's function, gives us the solution for the semi-homogeneous problem

$$
L_{n} y(x)=\sigma(x) \quad \text { for } x \in I, \quad U_{i}(y)=0 \quad \text { for } i=1,2, \ldots, m \text { and } m \leq n
$$

where $\sigma(x)$ is a continuous real function. In fact,

$$
y(x)=\int_{x_{1}}^{x_{2}} G_{n}(x, s) \sigma(s) d s \quad \text { for } x \in I
$$

is a solution of problem (1.15) [25].

In 1975, Das and Vatsala [10] obtained the following results for the (2n)th order problem:

$$
\begin{aligned}
& y^{(2 n)}=(-1)^{n-1} h(x), \\
& y^{(k)}\left(x_{1}\right)=y^{(k)}\left(x_{2}\right)=0 \text { for } k=0,1, \ldots, n-1,
\end{aligned}
$$

where $n \in \mathbb{N}, x_{1}, x_{2}$ are real numbers with $x_{1}<x_{2}$, and $y(x) \neq 0$ for all $x \in\left(x_{1}, x_{2}\right)$.

Lemma A ([10, Lemma 2.1]) Let $n \in \mathbb{N}$. Then the following identity

$$
\begin{aligned}
& {\left[\frac{\left(x-x_{1}\right)\left(x_{2}-s\right)}{x_{2}-x_{1}}\right]^{n} \sum_{j=0}^{n-1}\left(\begin{array}{c}
n-1+j \\
j
\end{array}\right)(s-x)^{n-j-1}\left[\frac{\left(s-x_{1}\right)\left(x_{2}-x\right)}{x_{2}-x_{1}}\right]^{j}} \\
& -(-1)^{n-1}(x-s)^{2 n-1}
\end{aligned}
$$




$$
=\left[\frac{\left(s-x_{1}\right)\left(x_{2}-x\right)}{x_{2}-x_{1}}\right]^{n} \sum_{j=0}^{n-1}\left(\begin{array}{c}
n-1+j \\
j
\end{array}\right)(x-s)^{n-j-1}\left[\frac{\left(x-x_{1}\right)\left(x_{2}-s\right)}{x_{2}-x_{1}}\right]^{j}
$$

holds.

Lemma B ([10, Theorem 2.1]) If $y(x)$ is a solution (not identically zero) on $\left[x_{1}, x_{2}\right]$ for problem (1.17)-(1.18), then

$$
y(x)=\int_{x_{1}}^{x_{2}} G_{2 n}(x, s) h(s) d s
$$

holds, where

$$
\begin{aligned}
G_{2 n}(x, s)= & \frac{1}{(2 n-1) !} \\
& \times \begin{cases}{\left[\frac{\left(x-x_{1}\right)\left(x_{2}-s\right)}{x_{2}-x_{1}}\right]^{n} \sum_{j=0}^{n-1}\left(\begin{array}{c}
n-1+j \\
j
\end{array}\right)(s-x)^{n-j-1}\left[\frac{\left(s-x_{1}\right)\left(x_{2}-x\right)}{x_{2}-x_{1}}\right]^{j} ;} & x \leq s \leq x_{2}, \\
{\left[\frac{\left(s-x_{1}\right)\left(x_{2}-x\right)}{x_{2}-x_{1}}\right]^{n} \sum_{j=0}^{n-1}\left(\begin{array}{c}
n-1+j \\
j
\end{array}\right)(x-s)^{n-j-1}\left[\frac{\left(x-x_{1}\right)\left(x_{2}-s\right)}{x_{2}-x_{1}}\right]^{j} ;} & x_{1} \leq s \leq x .\end{cases}
\end{aligned}
$$

It is easy to see that from Lemma $A$ and Lemma $B$ that we have the symmetric property of Green's function $G_{2 n}(x, s)$, i.e., $G_{2 n}(x, s)=G_{2 n}(s, x)$ for all $x, s \in\left[x_{1}, x_{2}\right]$.

Theorem A ([10, Theorem 3.1]) If $r \in C([0, \infty), \mathbb{R})$ and $y(x)$ is a solution (not identically zero) on $\left[x_{1}, x_{2}\right]$ for the problem

$$
y^{(2 n)}+(-1)^{n-1} r(x) y=0
$$

satisfying conditions (1.18), then the inequality

$$
(2 n-1)[(n-1) !]^{2}\left(x_{2}-x_{1}\right)^{2 n-1} \leq \int_{x_{1}}^{x_{2}}\left[\left(s-x_{1}\right)\left(x_{2}-s\right)\right]^{2 n-1} r^{+}(s) d s
$$

holds, where $r^{+}(x)=\max \{r(x), 0\}$.

In 2016, Dhar and Kong [13] obtained the following results for the $(2 n+1)$ th order problem:

$$
\begin{aligned}
& y^{(2 n+1)}+(-1)^{n-1} r(x) y=0, \\
& y^{(k+1)}\left(x_{1}\right)=y^{(k+1)}\left(x_{2}\right)=0 \quad \text { for } k=0,1, \ldots, n-1, \\
& y(c)=0 \quad \text { for } c \in\left[x_{1}, x_{2}\right],
\end{aligned}
$$

where $n \in \mathbb{N}, x_{1}, x_{2}$ are real numbers with $x_{1}<x_{2}$, and $y(x) \neq 0$ for all $x \in\left[x_{1}, x_{2}\right]-\{c\}$.

Theorem $\mathbf{B}$ ([13, Theorem 2.1]) If $r \in C([0, \infty), \mathbb{R})$ and $y(x)$ is a solution (not identically zero) on $\left[x_{1}, x_{2}\right]$ for problem (1.24)-(1.26), then the inequality

$$
\frac{2^{2 n}(2 n-1) !}{S_{n}\left(x_{2}-x_{1}\right)^{2 n}}<\int_{x_{1}}^{x_{2}}|r(s)| d s
$$


holds, where

$$
S_{n}=\sum_{j=0}^{n-1} \sum_{k=0}^{j} 2^{2 k-2 j}\left(\begin{array}{c}
n-1+j \\
j
\end{array}\right)\left(\begin{array}{l}
j \\
k
\end{array}\right) B(n+1, n+k-j),
$$

$B(\alpha, \beta)=\int_{0}^{1} s^{\alpha-1}(1-s)^{\beta-1} d s$ is the beta function for $\alpha, \beta>0$.

Theorem C ([13, Theorem 2.2]) Assume that $r \in C([0, \infty), \mathbb{R})$ and $y(x)$ is a solution (not identically zero) on $\left[x_{1}, x_{2}\right]$ for problem (1.24)-(1.25).

(a) Suppose $y(c)=0$ for $c \in\left(x_{1}, x_{2}\right)$ and $y(x) \neq 0$ for $x \in\left[x_{1}, x_{2}\right]-\{c\}$. Then one of the inequalities given below holds:

(i) $\frac{2^{2 n}(2 n-1) !}{S_{n}\left(x_{2}-x_{1}\right)^{2 n}}<\int_{x_{1}}^{x_{2}} r^{-}(s) d s$,

(ii) $\frac{2^{2 n}(2 n-1) !}{S_{n}\left(x_{2}-x_{1}\right)^{2 n}}<\int_{x_{1}}^{x_{2}} r^{+}(s) d s$,

(iii) $\frac{2^{2 n}(2 n-1) !}{S_{n}\left(x_{2}-x_{1}\right)^{2 n}}<\int_{x_{1}}^{c} r^{-}(s) d s+\int_{c}^{x_{2}} r^{+}(s) d s$,

where $S_{n}$ is given in (1.28) and

$$
r^{\mp}(x)=\max \{\mp r(x), 0\} .
$$

(b) Suppose $y\left(x_{1}\right)=0$ and $y(x) \neq 0$ for $x \in\left(x_{1}, x_{2}\right]$. Then the inequality

$$
\frac{2^{2 n}(2 n-1) !}{S_{n}\left(x_{2}-x_{1}\right)^{2 n}}<\int_{x_{1}}^{x_{2}} r^{+}(s) d s
$$

holds, where $S_{n}$ and $r^{+}(x)$ are given in (1.28) and (1.29), respectively.

(c) Suppose $y\left(x_{2}\right)=0$ and $y(x) \neq 0$ for $x \in\left[x_{1}, x_{2}\right)$. Then the inequality

$$
\frac{2^{2 n}(2 n-1) !}{S_{n}\left(x_{2}-x_{1}\right)^{2 n}}<\int_{x_{1}}^{x_{2}} r^{-}(s) d s
$$

holds, where $S_{n}$ and $r^{-}(x)$ are given in (1.28) and (1.29), respectively.

In this article, we investigate a new Lyapunov-type inequality for BVP of order $(2 n+1)$ given in (1.1)-(1.3). Firstly, we construct the Green's function for the same problem. And then, by bounding the Green's function, we obtain new Lyapunov-type inequalities for problem (1.1)-(1.3). Finally, we give some applications of the obtained inequalities.

\section{Main results}

In the following result, we construct Green's function for the $(2 n+1)$ th order differential equation

$$
y^{(2 n+1)}=(-1)^{n-1} g(x)
$$

with the boundary conditions (1.2)-(1.3) inspired by Das and Vatsala [10].

Lemma 2.1 If $y(x)$ is a solution (not identically zero) of problem (2.1) with (1.2)-(1.3), then

$$
y(x)=\int_{x_{1}}^{x_{2}} G_{2 n+1}(x, s) g(s) d s
$$


holds, where

$$
\begin{aligned}
G_{2 n+1}(x, s)= & \frac{1}{(2 n) !} \\
& \times\left\{\begin{array}{c}
{\left[\frac{\left(x-x_{1}\right)\left(x_{2}-s\right)}{x_{2}-x_{1}}\right]^{n} \sum_{j=0}^{n-1}\left(\begin{array}{c}
n-1+j \\
j
\end{array}\right)(s-x)^{n-j}\left[\frac{\left(s-x_{1}\right)\left(x_{2}-x\right)}{x_{2}-x_{1}}\right]^{j} ;} \\
{\left[\frac{\left(x-x_{1}\right)\left(x_{2}-s\right)}{x_{2}-x_{1}}\right]^{n} \sum_{j=0}^{n-1}\left(\begin{array}{c}
n-1+j \\
j
\end{array}\right)(s-x)^{n-j}\left[\frac{\left(s-x_{1}\right)\left(x_{2}-x\right)}{x_{2}-x_{1}}\right]^{j}+\frac{(x-s)^{2 n}}{(2 n) !} ;} \\
x_{1} \leq x .
\end{array}\right.
\end{aligned}
$$

Proof First of all, we show that the function $G_{2 n+1}(x, s)$ satisfies all the conditions of Definition A. Conditions $\left(G_{1}\right)-\left(G_{3}\right)$ and $\left(G_{5}\right)$ are obviously satisfied. It is enough to show that the function $G_{2 n+1}(x, s)$ satisfies conditions $\left(G_{4}\right)$ and $\left(G_{6}\right)$.

Now, we show that the function $G_{2 n+1}(x, s)$ satisfies condition $\left(G_{4}\right)$. Note that the function $G_{2 n+1}(x, s)$ has the same terms except for $\frac{(x-s)^{2 n}}{(2 n) !}$ for $x \leq s$ and $s \leq x$. Deriving $2 n$ times the function $G_{2 n+1}(x, s)$ with respect to $x$, it is easy to see that we have

$$
\frac{\partial^{2 n} G_{2 n+1}}{\partial x^{2 n}}\left(x, x^{+}\right)-\frac{\partial^{2 n} G_{2 n+1}}{\partial x^{2 n}}\left(x, x^{-}\right)=-1 .
$$

Therefore, the function $G_{2 n+1}(x, s)$ satisfies condition $\left(G_{4}\right)$.

Now, we show that function $G_{2 n+1}(x, s)$ satisfies condition $\left(G_{6}\right)$. For $x \leq s<x_{2}$, in the derivative of the multiplication of the functions $\left[\frac{\left(x-x_{1}\right)\left(x_{2}-s\right)}{x_{2}-x_{1}}\right]^{n}$ and $\sum_{j=0}^{n-1}\left(\begin{array}{c}n-1+j \\ j\end{array}\right)(s-$ $x)^{n-j-1}\left[\frac{\left(s-x_{1}\right)\left(x_{2}-x\right)}{x_{2}-x_{1}}\right]^{j}$, one term always includes $\left(x-x_{1}\right)^{k}$ for $k=1,2, \ldots, n$, which is zero at $x=x_{1}$. Deriving $n$ times other term and taking $x=x_{1}$, we get

$$
\left.\frac{\partial^{n} G_{2 n+1}(x, s)}{\partial x^{n}}\right|_{x=x_{1}}=n !\left[\frac{\left(s-x_{1}\right)\left(x_{2}-s\right)}{x_{2}-x_{1}}\right]^{n} \sum_{j=0}^{n-1}\left(\begin{array}{c}
n-1+j \\
j
\end{array}\right) .
$$

Similarly, for $x_{1}<s \leq x$, we have

$$
\left.\frac{\partial^{n} G_{2 n+1}(x, s)}{\partial x^{n}}\right|_{x=x_{2}}=(-1)^{n-1} n !\left[\frac{\left(s-x_{1}\right)\left(x_{2}-s\right)}{x_{2}-x_{1}}\right]^{n} \sum_{j=0}^{n-1}\left(\begin{array}{c}
n-1+j \\
j
\end{array}\right) .
$$

Thus, we get

$$
\left.(-1)^{n-1} \frac{\partial^{n} G_{2 n+1}(x, s)}{\partial x^{n}}\right|_{x=x_{1}}=\left.\frac{\partial^{n} G_{2 n+1}(x, s)}{\partial x^{n}}\right|_{x=x_{2}} .
$$

Therefore, the function $G_{2 n+1}(x, s)$ satisfies condition $\left(G_{6}\right)$.

Thus, the function $G_{2 n+1}(x, s)$ satisfies all the conditions of Definition A. It is said that the function $G_{2 n+1}(x, s)$ is a Green's function for problem (2.1) with (1.2)-(1.3). Then, from (1.15)-(1.16), we obtain (2.2) for the problem.

Remark 2.1 We note that from the boundary condition $(-1)^{n-1} y^{(n)}\left(x_{1}\right)=y^{(n)}\left(x_{2}\right)$ in (1.3) is not equal to zero from (2.5) and (2.6) since condition $\left(G_{6}\right)$ is satisfied for $s \in\left(x_{1}, x_{2}\right)$.

Now, we prove the following lemma which is used by the anti-symmetric property of Green's function $G_{2 n+1}(x, s)$, i.e., $G_{2 n+1}(x, s)=-G_{2 n+1}(s, x)$ for all $x, s \in\left[x_{1}, x_{2}\right]$. 
Lemma 2.2 Let $n \in \mathbb{N}$. Then the following identity

$$
\begin{aligned}
& {\left[\frac{\left(x-x_{1}\right)\left(x_{2}-s\right)}{x_{2}-x_{1}}\right]^{n} \sum_{j=0}^{n-1}\left(\begin{array}{c}
n-1+j \\
j
\end{array}\right)(s-x)^{n-j}\left[\frac{\left(s-x_{1}\right)\left(x_{2}-x\right)}{x_{2}-x_{1}}\right]^{j}} \\
& +(-1)^{n-1}(x-s)^{2 n} \\
& =-\left[\frac{\left(s-x_{1}\right)\left(x_{2}-x\right)}{x_{2}-x_{1}}\right]^{n} \sum_{j=0}^{n-1}\left(\begin{array}{c}
n-1+j \\
j
\end{array}\right)(x-s)^{n-j} \\
& \quad \times\left[\frac{\left(x-x_{1}\right)\left(x_{2}-s\right)}{x_{2}-x_{1}}\right]^{j}
\end{aligned}
$$

holds.

Proof We use the induction principle. It is obvious that the equality is true when $n=1$. If so, then we have

$$
\frac{\left(x-x_{1}\right)\left(x_{2}-s\right)(s-x)}{x_{2}-x_{1}}+(x-s)^{2}=-\frac{\left(s-x_{1}\right)\left(x_{2}-x\right)(x-s)}{x_{2}-x_{1}} .
$$

In the next step, we assume that the equality is true for $n=m$, and we prove that it is true for $n=m+1$. By using the left-hand side of (2.8) with $n=m+1$ and (2.9), we have

$$
\begin{aligned}
& {\left[\frac{\left(x-x_{1}\right)\left(x_{2}-s\right)}{x_{2}-x_{1}}\right]^{m}\left\{\sum_{j=0}^{m}\left(\begin{array}{c}
m+j \\
j
\end{array}\right)(s-x)^{m+1-j}\left[\frac{\left(s-x_{1}\right)\left(x_{2}-x\right)}{x_{2}-x_{1}}\right]^{j+1}\right.} \\
& \left.-\sum_{j=0}^{m}\left(\begin{array}{c}
m+j \\
j
\end{array}\right)(s-x)^{m+2-j}\left[\frac{\left(s-x_{1}\right)\left(x_{2}-x\right)}{x_{2}-x_{1}}\right]^{j}\right\}+(-1)^{m}(x-s)^{2 m+2} \\
& =\left[\frac{\left(x-x_{1}\right)\left(x_{2}-s\right)}{x_{2}-x_{1}}\right]^{m}\left\{\left(\begin{array}{c}
2 m \\
m
\end{array}\right)(s-x)\left[\frac{\left(s-x_{1}\right)\left(x_{2}-x\right)}{x_{2}-x_{1}}\right]^{m+1}\right. \\
& \left.-\sum_{j=0}^{m}\left(\begin{array}{c}
m-1+j \\
j
\end{array}\right)(s-x)^{m+2-j}\left[\frac{\left(s-x_{1}\right)\left(x_{2}-x\right)}{x_{2}-x_{1}}\right]^{j}\right\}+(-1)^{m}(x-s)^{2 m+2} \\
& =\left[\frac{\left(x-x_{1}\right)\left(x_{2}-s\right)}{x_{2}-x_{1}}\right]^{m}\left\{\left(\begin{array}{c}
2 m \\
m
\end{array}\right)(s-x)\left[\frac{\left(s-x_{1}\right)\left(x_{2}-x\right)}{x_{2}-x_{1}}\right]^{m+1}\right. \\
& \left.-\left(\begin{array}{c}
2 m-1 \\
m
\end{array}\right)(s-x)^{2}\left[\frac{\left(s-x_{1}\right)\left(x_{2}-x\right)}{x_{2}-x_{1}}\right]^{m}\right\} \\
& +\left[\frac{\left(s-x_{1}\right)\left(x_{2}-x\right)}{x_{2}-x_{1}}\right]^{m} \sum_{j=0}^{m-1}\left(\begin{array}{c}
m-1+j \\
j
\end{array}\right)(x-s)^{m+2-j} \\
& \times\left[\frac{\left(x-x_{1}\right)\left(x_{2}-s\right)}{x_{2}-x_{1}}\right]^{j} .
\end{aligned}
$$

By using the formula

$$
\left(\begin{array}{c}
m-1+j \\
j
\end{array}\right)=\left(\begin{array}{c}
m+j \\
j
\end{array}\right)-\left(\begin{array}{c}
m-1+j \\
j-1
\end{array}\right)
$$


for $j=1, \ldots, m-1$ in $(2.10)$, we have

$$
\begin{aligned}
& -\left[\frac{\left(s-x_{1}\right)\left(x_{2}-x\right)}{x_{2}-x_{1}}\right]^{m+1}\left\{\left(\begin{array}{c}
2 m \\
m
\end{array}\right)(x-s)\left[\frac{\left(x-x_{1}\right)\left(x_{2}-s\right)}{x_{2}-x_{1}}\right]^{m}\right. \\
& \left.+\sum_{j=0}^{m-1}\left(\begin{array}{c}
m+j \\
j
\end{array}\right)(x-s)^{m+1-j}\left[\frac{\left(x_{2}-s\right)\left(x-x_{1}\right)}{x_{2}-x_{1}}\right]^{j}\right\}
\end{aligned}
$$

which is equal to

$$
-\left[\frac{\left(s-x_{1}\right)\left(x_{2}-x\right)}{x_{2}-x_{1}}\right]^{m+1} \sum_{j=0}^{m}\left(\begin{array}{c}
m+j \\
j
\end{array}\right)(x-s)^{m+1-j}\left[\frac{\left(x-x_{1}\right)\left(x_{2}-s\right)}{x_{2}-x_{1}}\right]^{j} .
$$

This completes the proof.

Remark 2.2 From Lemma 2.2, it is easy to see that the Green's function $G_{2 n+1}(x, s)$ has got the anti-symmetric property $G_{2 n+1}(x, s)=-G_{2 n+1}(s, x)$ for all $x, s \in\left[x_{1}, x_{2}\right]$. Then, we can rewrite (2.3) as follows:

$$
\begin{aligned}
& G_{2 n+1}(x, s) \\
& \quad=\frac{1}{(2 n) !} \begin{cases}{\left[\frac{\left(x-x_{1}\right)\left(x_{2}-s\right)}{x_{2}-x_{1}}\right]^{n} \sum_{j=0}^{n-1}\left(\begin{array}{c}
n-1+j \\
j
\end{array}\right)(s-x)^{n-j}\left[\frac{\left(s-x_{1}\right)\left(x_{2}-x\right)}{x_{2}-x_{1}}\right]^{j} ;} & x \leq s \leq x_{2}, \\
-\left[\frac{\left(s-x_{1}\right)\left(x_{2}-x\right)}{x_{2}-x_{1}}\right]^{n} \sum_{j=0}^{n-1}\left(\begin{array}{c}
n-1+j \\
j
\end{array}\right)(x-s)^{n-j}\left[\frac{\left(x-x_{1}\right)\left(x_{2}-s\right)}{x_{2}-x_{1}}\right]^{j} ; & x_{1} \leq s \leq x .\end{cases}
\end{aligned}
$$

In the proof of Lemma 2.2, for $x \geq s$, (2.6) can also be obtained by using (2.14). Moreover, we have $G_{2 n+1}(x, s)=\frac{s-x}{2 n} G_{2 n}(x, s)$ for all $x, s \in\left[x_{1}, x_{2}\right]$.

Theorem 2.1 If $y(x)$ is a solution (not identically zero) on $\left[x_{1}, x_{2}\right]$ for problem (1.1)-(1.3), then the inequality

$$
1 \leq \int_{x_{1}}^{x_{2}}\left|G_{2 n+1}\left(x_{*}, s\right)\right||r(s)| d s
$$

holds, where $G_{2 n+1}(x, s)$ is as defined in (2.3) or (2.14), and $\left|y\left(x_{*}\right)\right|=\max \left\{|y(x)|: x_{1} \leq x \leq x_{2}\right\}$.

Proof Let $y^{(k)}\left(x_{1}\right)=y^{(k)}\left(x_{2}\right)=0$ for $k=0,1, \ldots, n-1,(-1)^{n-1} y^{(n)}\left(x_{1}\right)=y^{(n)}\left(x_{2}\right)$ with $x_{1}<x_{2}$ and $y(x) \neq 0$ for all $x \in\left(x_{1}, x_{2}\right)$. Pick $x_{*} \in\left(x_{1}, x_{2}\right)$ so that $\left|y\left(x_{*}\right)\right|=\max \left\{|y(x)|: x_{1} \leq x \leq x_{2}\right\}$. From (1.1) and (2.2) with $g(x)=(-1)^{n} r(x) y(x)$, we obtain

$$
\left|y\left(x_{*}\right)\right| \leq \int_{x_{1}}^{x_{2}}\left|G_{2 n+1}\left(x_{*}, s\right)\right||r(s)||y(s)| d s,
$$

and hence

$$
\left|y\left(x_{*}\right)\right| \leq\left|y\left(x_{*}\right)\right| \int_{x_{1}}^{x_{2}}\left|G_{2 n+1}\left(x_{*}, s\right)\right||r(s)| d s .
$$

Dividing both sides by $\left|y\left(x_{*}\right)\right|$, we obtain inequality (2.15). 
It is clear that since $G_{2 n}(x, s) \leq G_{2 n}(s, s)$ for all $x, s \in\left[x_{1}, x_{2}\right]$ in the paper [10], we have the following inequalities:

$$
\left|G_{2 n+1}(x, s)\right| \leq \frac{x_{2}-s}{2 n} G_{2 n}(s, s) \text { for } x_{1} \leq s \leq x
$$

and

$$
G_{2 n+1}(x, s) \leq \frac{s-x_{1}}{2 n} G_{2 n}(s, s) \quad \text { for } x \leq s \leq x_{2}
$$

from Remark 2.2. Then we define

$$
G_{2 n+1}^{*}(s):= \begin{cases}\frac{x_{2}-s}{2 n} G_{2 n}(s, s) ; & s<\frac{x_{1}+x_{2}}{2} \\ \frac{s-x_{1}}{2 n} G_{2 n}(s, s) ; & s>\frac{x_{1}+x_{2}}{2} .\end{cases}
$$

By using inequalities (2.18)-(2.20), we obtain the following theorem and hence the proof is omitted.

Theorem 2.2 If $y(x)$ is a solution (not identically zero) on $\left[x_{1}, x_{2}\right]$ for problem (1.1)-(1.3), then the inequality

$$
1 \leq \int_{x_{1}}^{x_{2}}\left|G_{2 n+1}^{*}(s)\right||r(s)| d s
$$

holds, where $G_{2 n+1}^{*}(s)$ is as defined in (2.20).

Now, we find the maximum of the function $G_{2 n+1}(x, s)$ for $x \leq s \leq x_{2}$. Since

$$
\begin{aligned}
& \left(s-x_{1}\right)^{j}=\left(s-x+x-x_{1}\right)^{j}=\sum_{m=0}^{j}\left(\begin{array}{c}
j \\
m
\end{array}\right)\left(x-x_{1}\right)^{m}(s-x)^{j-m}, \\
& \left(x_{2}-x\right)^{j}=\left(x_{2}-s+s-x\right)^{j}=\sum_{k=0}^{j}\left(\begin{array}{l}
j \\
k
\end{array}\right)\left(x_{2}-s\right)^{k}(s-x)^{j-k},
\end{aligned}
$$

and

$$
G_{2 n+1}(x, s)=\sum_{j=0}^{n-1}\left(\begin{array}{c}
n-1+j \\
j
\end{array}\right) \frac{\left(x-x_{1}\right)^{n}\left(x_{2}-s\right)^{n}(s-x)^{n-j}\left(s-x_{1}\right)^{j}\left(x_{2}-x\right)^{j}}{(2 n) !\left(x_{2}-x_{1}\right)^{n+j}}
$$

for $x \leq s \leq x_{2}$, we get

$$
G_{2 n+1}(x, s)=\sum_{j=0}^{n-1} \sum_{k=0}^{j} \sum_{m=0}^{j}\left(\begin{array}{c}
n-1+j \\
j
\end{array}\right)\left(\begin{array}{l}
j \\
k
\end{array}\right)\left(\begin{array}{l}
j \\
m
\end{array}\right) \frac{H_{1}(x, s)}{(2 n) !\left(x_{2}-x_{1}\right)^{n+j}},
$$

where $H_{1}(x, s):=\left(x-x_{1}\right)^{n+m}\left(x_{2}-s\right)^{n+k}(s-x)^{n+j-m-k}$. The function $H_{1}(x, s)$ takes its maximum value at the point

$$
\left(x_{0}, s_{0}\right)=\left(\frac{x_{1}(2 n+j-m)+x_{2}(m+n)}{3 n+j}, \frac{x_{1}(k+n)+x_{2}(2 n+j-k)}{3 n+j}\right),
$$


and its maximum value is

$$
\begin{aligned}
& H_{1}\left(\frac{x_{1}(2 n+j-m)+x_{2}(m+n)}{3 n+j}, \frac{x_{1}(k+n)+x_{2}(2 n+j-k)}{3 n+j}\right) \\
& \quad=\frac{(k+n)^{k+n}(m+n)^{m+n}\left(x_{2}-x_{1}\right)^{j+3 n}}{(j+n-k-m)^{k+m-j-n}(j+3 n)^{j+3 n}} .
\end{aligned}
$$

Thus, we get

$$
G_{2 n+1}(x, s) \leq \frac{C_{n}\left(x_{2}-x_{1}\right)^{2 n}}{(2 n) !}
$$

where

$$
C_{n}:=\sum_{j=0}^{n-1} \sum_{k=0}^{j} \sum_{m=0}^{j}\left(\begin{array}{c}
n-1+j \\
j
\end{array}\right)\left(\begin{array}{c}
j \\
k
\end{array}\right)\left(\begin{array}{l}
j \\
m
\end{array}\right) \frac{(k+n)^{k+n}(m+n)^{m+n}}{(j+n-k-m)^{k+m-j-n}(j+3 n)^{j+3 n}}
$$

for $x \leq s \leq x_{2}$. Since $G_{2 n+1}(x, s)=-G_{2 n+1}(s, x)$ for all $x, s \in\left[x_{1}, x_{2}\right]$, we have

$$
\left|G_{2 n+1}(x, s)\right| \leq \frac{C_{n}\left(x_{2}-x_{1}\right)^{2 n}}{(2 n) !}
$$

for all $x, s \in\left[x_{1}, x_{2}\right]$.

Thus, by using the result obtained in (2.15), we get the following main theorem, and hence the proof is omitted.

Theorem 2.3 If $y(x)$ is a solution (not identically zero) on $\left[x_{1}, x_{2}\right]$ for problem (1.1)-(1.3), then the inequality

$$
\frac{(2 n) !}{C_{n}\left(x_{2}-x_{1}\right)^{2 n}} \leq \int_{x_{1}}^{x_{2}}|r(s)| d s
$$

holds, where $C_{n}$ is given in (2.29).

Remark 2.3 Note that if we take $n=1$ in Lemma 2.1, then

$$
y(x)=\int_{x_{1}}^{x_{2}} G_{3}(x, s) g(s) d s
$$

holds, where

$$
G_{3}(x, s)= \begin{cases}\frac{\left(x-x_{1}\right)\left(x_{2}-s\right)(s-x)}{2\left(x_{2}-x_{1}\right)} ; & x \leq s \leq x_{2} \\ -\frac{(x-s)\left(x_{2}-x\right)\left(s-x_{1}\right)}{2\left(x_{2}-x_{1}\right)} ; & x_{1} \leq s \leq x .\end{cases}
$$

Now, we find the absolute maximum of Green's function (2.33). Consider $G_{3}(x, s)=$ $\frac{\left(x-x_{1}\right)\left(x_{2}-s\right)(s-x)}{2\left(x_{2}-x_{1}\right)}$ for $x \leq s \leq x_{2} . G_{3}(x, s)$ takes its absolute maximum value at the point $\left(x_{0}, s_{0}\right)=\left(\frac{2 x_{1}+x_{2}}{3}, \frac{x_{1}+2 x_{2}}{3}\right)$, and its absolute maximum value is $G_{3}\left(\frac{2 x_{1}+x_{2}}{3}, \frac{x_{1}+2 x_{2}}{3}\right)=\frac{\left(x_{2}-x_{1}\right)^{2}}{54}$. Since $G_{3}(x, s)=-G_{3}(s, x)$ for all $x, s \in\left[x_{1}, x_{2}\right]$, we have

$$
\left|G_{3}(x, s)\right| \leq \frac{\left(x_{2}-x_{1}\right)^{2}}{54}
$$


for all $x, s \in\left[x_{1}, x_{2}\right]$. Thus, from (2.31) with $n=1$, we have the following Lyapunov-type inequality:

$$
\frac{54}{\left(x_{2}-x_{1}\right)^{2}} \leq \int_{x_{1}}^{x_{2}}|r(s)| d s
$$

We believe that constant 54 is the best possibility for problem (1.1)-(1.3) with $n=1$ in view of the fact that if constant 54 in the left-hand side of (2.35) cannot be replaced by any larger constant.

To extend oscillation criteria given below with the help of Lyapunov-type inequality, we can use an alternative way and use (2.31) (cf. [26]): $y^{\prime \prime}(x)$ and $y^{\prime \prime}(x) y^{-1}(x)$ are continuous for $x_{1} \leq x \leq x_{2}$, with $y\left(x_{1}\right)=y\left(x_{2}\right)=0$, then

$$
\frac{4}{x_{2}-x_{1}}<\int_{x_{1}}^{x_{2}}\left|y^{\prime \prime}(s) y^{-1}(s)\right| d s
$$

Thus, from (2.31), we get the following extension: If $y^{(2 n+1)}(x)$ and $y^{(2 n+1)}(x) y^{-1}(x)$ are continuous for $x_{1} \leq x \leq x_{2}$, with $y^{(k)}\left(x_{1}\right)=y^{(k)}\left(x_{2}\right)=0$ for $k=0,1, \ldots, n-1,(-1)^{n-1} y^{(n)}\left(x_{1}\right)=$ $y^{(n)}\left(x_{2}\right)$, then

$$
\frac{(2 n) !}{C_{n}\left(x_{2}-x_{1}\right)^{2 n}}<\int_{x_{1}}^{x_{2}}\left|y^{(2 n+1)}(s) y^{-1}(s)\right| d s,
$$

where $C_{n}$ is given in (2.29).

Finally, we consider another useful application of the Lyapunov-type inequality obtained in (2.31) for the eigenvalue problem

$$
y^{(2 n+1)}+\lambda h(x) y=0
$$

with the conditions in (1.2)-(1.3). Thus, if there exists a solution (not identically zero) $y(x)$ of problem (2.38), then the inequality

$$
\frac{(2 n) !}{C_{n}\left(x_{2}-x_{1}\right)^{2 n} \int_{x_{1}}^{x_{2}}|h(s)| d s}<|\lambda|
$$

holds, where $C_{n}$ is given in (2.29).

\section{Acknowledgements}

The authors would like to thank the anonymous referees for their valuable suggestions and comments.

Funding

Not applicable.

\section{Abbreviations}

Not applicable.

Availability of data and materials

Not applicable.

Ethics approval and consent to participate

The authors contributed to each part of this study. 


\section{Consent for publication}

The authors read and approved the final version of the manuscript.

\section{Authors' contributions}

All authors contributed equally to this paper. They read and approved the manuscript.

\section{Author details}

'Department of Mathematics, Faculty of Sciences, Gazi University, Ankara, Turkey. ${ }^{2}$ Department of Mathematics

Education, Faculty of Education, Gazi University, Ankara, Turkey.

\section{Publisher's Note}

Springer Nature remains neutral with regard to jurisdictional claims in published maps and institutional affiliations.

\section{Received: 17 January 2019 Accepted: 13 May 2019 Published online: 23 May 2019}

\section{References}

1. Liapunov, A.M.: Probleme general de la stabilite du mouvement. Ann. Fac. Sci. Univ. Toulouse 2, 203-407 (1907)

2. Hartman, P.: Ordinary Differential Equations. Birkhauser, Boston (1982)

3. Kelley, W.G., Peterson, A.C.: The Theory of Differential Equations, Classical and Qualitative. Springer, New York (2010)

4. Agarwal, R.P., Özbekler, A.: Lyapunov type inequalities for $n$th order forced differential equations with mixed nonlinearities. Commun. Pure Appl. Anal. 15, 2281-2300 (2016)

5. Agarwal, R.P., Özbekler, A.: Lyapunov type inequalities for even order differential equations with mixed nonlinearities. J. Inequal. Appl. 2015, 142 (2015)

6. Aktaş, M.F., Çakmak, D.: Lyapunov-type inequalities for third-order linear differential equations. Electron. J. Differ. Equ. 2017, 139 (2017)

7. Aktaş, M.F., Çakmak, D., Tiryaki, A.: On the Lyapunov-type inequalities of a three-point boundary value problem for third order linear differential equations. Appl. Math. Lett. 45, 1-6 (2015)

8. Beesack, P.R.: On the Green's function of an n-point boundary value problem. Pac. J. Math. 12, 801-812 (1962)

9. Çakmak, D.: Lyapunov-type integral inequalities for certain higher order differential equations. Appl. Math. Comput. 216, 368-373 (2010)

10. Das, K.M., Vatsala, A.S.: Green's function for $n-n$ boundary value problem and an analogue of Hartman's result. J. Math. Anal. Appl. 51, 670-677 (1975)

11. Das, K.M., Vatsala, A.S.: On Green's function of an $n$-point boundary value problem. Trans. Am. Math. Soc. 182, 469-480 (1973)

12. Dhar, S., Kong, Q.: Lyapunov-type inequalities for third-order linear differential equations. Math. Inequal. Appl. 19, 297-312 (2016)

13. Dhar, S., Kong, Q.: Lyapunov-type inequalities for odd order linear differential equations. Electron. J. Differ. Equ. 2016, $243(2016)$

14. He, X., Tang, X.H.: Lyapunov-type inequalities for even order differential equations. Commun. Pure Appl. Anal. 11, 465-473 (2012)

15. Pachpatte, B.G.: On Lyapunov-type inequalities for certain higher order differential equations. J. Math. Anal. Appl. 195, 527-536 (1995)

16. Panigrahi, S.: Lyapunov-type integral inequalities for certain higher order differential equations. Electron. J. Differ. Equ. 2009, 4 (2009)

17. Parhi, N., Panigrahi, S.: Disfocality and Liapunov-type inequalities for third-order equations. Appl. Math. Lett. 16, 227-233 (2003)

18. Parhi, N., Panigrahi, S.: Liapunov-type inequality for higher order differential equations. Math. Slovaca 52, 31-46 (2002)

19. Parhi, N., Panigrahi, S.: Liapunov-type inequality for delay-differential equations of third order. Czechoslov. Math. J. 52, 385-399 (2002)

20. Parhi, N., Panigrahi, S.: On Liapunov-type inequality for third-order differential equations. J. Math. Anal. Appl. 233, 445-460 (1999)

21. Yang, X., Kim, Y., Lo, K.: A Lyapunov-type inequality for a two-term even-order differential equation. Math. Inequal. Appl. 15, 525-528 (2012)

22. Yang, X., Lo, K.: Lyapunov-type inequality for a class of even-order differential equations. Appl. Math. Comput. 215 3884-3890 (2010)

23. Yang, X., Kim, Y., Lo, K.: Lyapunov-type inequality for a class of odd-order differential equations. J. Comput. Appl. Math. 234, 2962-2968 (2010)

24. Yang, X.: On Liapunov-type inequality for certain higher-order differential equations. Appl. Math. Comput. 134, 307-317 (2003)

25. Cabada, A., Cid, J.A., Maquez-Villamarin, B.: Computation of Green's functions for boundary value problems with mathematica. Appl. Math. Comput. 219, 1919-1936 (2012)

26. Borg, G.: On a Liapounoff criterion of stability. Am. J. Math. 71, 67-70 (1949) 